\title{
Urinary NGAL to define AKI in asphyxiated infants
}

\author{
Stuart L. Goldstein
}

Received: 3 January 2015 /Revised: 3 January 2015 / Accepted: 5 January 2015 /Published online: 3 February 2015

(C) IPNA 2015

\begin{abstract}
Acute kidney injury (AKI) is independently associated with poor outcomes in the critically ill patient. The standard kidney function biomarker, serum creatinine, shows a demonstrable rise in concentration many hours to days after insult to the kidney. Thus, creatinine-based AKI diagnosis is likely delayed, rendering treatments to mitigate or prevent AKI ineffective. Neonatal AKI is further confounded by the fact that infant serum creatinine concentrations reflect maternal levels. The past 15 years has seen a massive research effort to identify early damage markers of AKI, with the hope that earlier "sub-clinical" AKI diagnosis can lead to earlier initiation of AKI treatment, or to adjustment of care to mitigate the adverse effects of AKI until renal function recovery occurs. One of the most promising urinary AKI biomarkers, neutrophil gelatinase associated lipocalin (NGAL), has repeatedly performed well to predict AKI in many pediatric populations, including those post-cardiac surgery, critically ill mechanically ventilated children and children arriving to the emergency department. The study reported by Admani et al. uses NGAL not only to predict serum creatinine-based AKI, but also to define AKI to associate a Day 1 NGAL concentration above a specific threshold with clinical outcomes.
\end{abstract}

Keywords Acute kidney injury · Early damage markers . $\mathrm{NGAL} \cdot$ Term infants $\cdot$ Asphyxiated infants

\section{Introduction}

Admani and colleagues [1] report on a prospective cohort evaluation of acute kidney injury (AKI)-associated outcomes

\section{S. L. Goldstein $(\triangle)$}

Center for Acute Care Nephrology, Cincinnati Children's Hospital

Medical Center, 3333 Burnet Avenue, MLC 7022,

Cincinnati, OH 45229, USA

e-mail: stuart.goldstein@cchmc.org in 108 asphyxiated newborns at two tertiary care centers in Nairobi, Africa. In their study, the authors use the novel urinary AKI biomarker, neutrophil gelatinase associated lipocalin (NGAL) not only to predict the development of serum creatinine ( $\mathrm{SCr}$ )-based $\mathrm{AKI}$, but also to define $\mathrm{AKI}$, establishing an NGAL threshold concentration $(250 \mathrm{ng} / \mathrm{ml})$ to demonstrate that NGAL-AKI itself is associated with poor outcomes. This study adds to the growing evidence that subclinical AKI is an important entity that portends poor prognosis and that NGAL may be an important clinical marker in a previously unstudied population. This commentary will focus on the epidemic of AKI in the pediatric population, the further difficulty in defining AKI in infants and the work that still needs to be done to integrate novel AKI biomarkers into clinical practice with the hope of improving patient outcomes.

\section{The impact of AKI in children}

The past 20 years has seen a shift in our understanding of the significant impact AKI has on short- and long-term outcomes in children. Prior to the 1990 s, critically ill patients were thought to be dying "with" and not "from" AKI. The first critical step to advance the field was the observation that AKI more often results from another systemic illness (e.g. sepsis, cardiac surgery, liver disease) or treatment of that illness (e.g. nephrotoxic antibiotics), rather than the primary kidney injury itself [2-4]. The next advancement was the development of a standardized multidimensional AKI classification system; this was first validated in adults [5], then modified for children [6] and ultimately harmonized across all patient populations - except for newborn children and infants [7]. Since that time, the preponderance of epidemiological data support the concept that AKI is associated with mortality and morbidity in children, independent of underlying comorbidities and severity of patient illness $[6,8-10]$. As a 
result, the importance of AKI and its impact across the pediatric disease spectrum has become manifest and is now taken into account not only by nephrologists, but by all pediatric sub-specialists. These developments have led to the field of critical care nephrology being born.

\section{Infants and AKI-the uncharted clinical research population}

As noted above, the modern AKI classification systems have not, for the most part, been applied to neonates, term newborn children and infants. The notable exception is the post-cardiac surgery population, where the incidence of AKI (defined as a $50 \% \mathrm{SCr}$ concentration increase above baseline) is approximately $40 \%$ [10-12]. The pediatric cardiac surgery population is an appealing target population for an AKI study since the timing of AKI is known (cardiopulmonary bypass), and the population has little co-morbidity. Aside from the cardiac surgery population, neonatal AKI research is challenged by a number of issues, including lack of systematic $\mathrm{SCr}$ surveillance in neonates and the contribution of maternal SCr to newborn $\mathrm{SCr}$ in the early post-natal period [13]. This led to the National Institute of Diabetes and Digestive and Kidney Disease convening a 1-day workshop in 2013 to highlight challenges facing neonatal AKI clinical research (http:// www.niddk.nih.gov/news/events-calendar/Pages/neonatalacute-kidney-injury-workshop.aspx). While white papers from this Workshop will be forthcoming, the recent high profile of neonatal AKI provides some encouragement that research in this area will be accelerated.

\section{$\mathrm{SCr}$ as a late functional marker of AKI}

The poor outcomes associated with $\mathrm{SCr}$ increases that were previously considered to be "modest" likely result from the fact that $\mathrm{SCr}$ is a late marker of kidney injury, analogous to electroencephalogram changes in the temporal evolution of the acute coronary syndrome. The past 15 years has also seen a huge research effort to find earlier, more sensitive markers of AKI to predict AKI development and severity. While a thorough review is beyond the scope of this commentary, and excellent reviews have been published recently $[14,15]$, novel AKI biomarkers have performed very well to predict AKI development in children after cardiac surgery [16], in critically ill children [17] and even in pre-term neonates [18]. Of these, urinary NGAL has been the most often studied biomarker in the pediatric population. Recent pooled analysis data also demonstrate that adults and children with "creatinine-negative, NGAL-positive" AKI have similarly poor outcomes as patients with
“creatinine-positive/NGAL-positive" AKI, suggesting that sub-clinical AKI as evidenced by tubular damage is an entity that needs to be brought to the forefront $[19,20]$, as does the concept of that the AKI definition "might need reassessment". In addition, the combination of functional and damage AKI biomarkers has just been shown to predict, with great precision and better than $\mathrm{SCr}$ itself, just which children after cardiac surgery will develop transient versus persistent AKI [21]. Thus, the pediatric AKI field may be on the verge of integrating novel and functional biomarkers into the correct clinical context to identify which patients will have severe AKI and worse outcomes.

\section{How does the current study by Amani et al. fit into the pediatric AKI research experience?}

The study by Admani et al. [1] provides some novel information to the field. Similar to the cardiac surgery research paradigm, Admani et al. [1] leveraged the known perinatal timing of and exposure to asphyxia to prospectively assess for AKI development by $\mathrm{SCr}$, as well as to assess the ability of NGAL to predict SCr-based AKI and to define AKI itself. These authors demonstrate that newborns' NGAL level has a moderate discriminatory power to predict SCr-based AKI with an AUC of 0.72 . Importantly, the negative predictive value in their study was very high $(95 \%)$, such that a NGAL level below their threshold was a strong predictor that AKI would not develop. This is of fundamental importance for the use of biomarkers, as capricious use with low sensitivity will lead to unnecessary interventions and resource utilization [22]. Further, the authors go on to demonstrate that a Day 1 NGAL concentration of $>250 \mathrm{ng} / \mathrm{ml}$ is also predictive of the development of hypoxic ischemic encephalopathy and patient mortality. As noted above, elevated NGAL may be a manifestation of a sub-clinical AKI portending worse systemic disease. While only speculative, we and others have shown that fluid overload may dilute SCr and mask SCr-based AKI detection $[23,24]$. One previous study used urinary NGAL — and not $\mathrm{SCr}$ - as the AKI definition in an AKI prevention study using fenoldopam versus placebo after cardiac surgery [25]. Given the confounding of maternal $\mathrm{SCr}$, the low muscle mass of newborns and the developmental increase in GFR seen over the first year of life, the ability to detect AKI independent of $\mathrm{SCr}$ would be a major boon to pediatric care. Admani and colleagues [1] should be commended for extrapolating the previous AKI work into a new patient population, correctly focusing on a subset of the newborn population with an identifiable time of insult and high morbidity associated with AKI [26]. Further work should build upon theirs to ascertain whether or not we can define AKI, as they have, by novel AKI damage biomarkers in other newborn populations. 


\section{References}

1. Essajee F, Were F, Admani B (2014) Urine neutrophil gelatinase associated lipocalin in asphyxiated neonates: A prospective cohort study. Pediatr Nephrol doi:10.1007/s00467-014-3035-9

2. Hui-Stickle S, Brewer ED, Goldstein SL (2005) Pediatric ARF epidemiology at a tertiary care center from 1999 to 2001. Am J Kidney Dis 45:96-101

3. Vachvanichsanong P, Dissaneewate P, Lim A, McNeil E (2006) Childhood acute renal failure: 22-year experience in a university hospital in southern Thailand. Pediatrics 118:e786-e791

4. Williams DM, Sreedhar SS, Mickell JJ, Chan JC (2002) Acute kidney failure: a pediatric experience over 20 years. Arch Pediatr Adolesc Med 156:893-900

5. Bellomo R, Ronco C, Kellum JA, Mehta RL, Palevsky P (2004) Acute renal failure - definition, outcome measures, animal models, fluid therapy and information technology needs: the Second International Consensus Conference of the Acute Dialysis Quality Initiative (ADQI) Group. Crit Care 8:R204-R212

6. Akcan-Arikan A, Zappitelli M, Loftis LL, Washburn KK, Jefferson LS, Goldstein SL (2007) Modified RIFLE criteria in critically ill children with acute kidney injury. Kidney Int 71:1028-1035

7. Kidney Disease: Improving Global Outcomes (KDIGO) Acute Kidney Injury Work Group (2012) Kidney Disease: Improving Global Outcomes (KDIGO) KDIGO Clinical Practice Guideline for Acute Kidney Injury. Kidney Int Suppl:1-138

8. Plotz FB, Bouma AB, van Wijk JA, Kneyber MC, Bokenkamp A (2008) Pediatric acute kidney injury in the ICU: an independent evaluation of pRIFLE criteria. Intensive Care Med 34:1713-1717

9. Slater MB, Anand V, Uleryk EM, Parshuram CS (2012) A systematic review of RIFLE criteria in children, and its application and association with measures of mortality and morbidity. Kidney Int 81:791798

10. Blinder JJ, Goldstein SL, Lee VV, Baycroft A, Fraser CD, Nelson D, Jefferies JL (2012) Congenital heart surgery in infants: Effects of acute kidney injury on outcomes. J Thorac Cardiovasc Surg 143: 368-374

11. Zappitelli M, Bernier PL, Saczkowski RS, Tchervenkov CI, Gottesman R, Dancea A, Hyder A, Alkandari O (2009) A small post-operative rise in serum creatinine predicts acute kidney injury in children undergoing cardiac surgery. Kidney Int 76:885-892

12. Morgan CJ, Zappitelli M, Robertson CM, Alton GY, Sauve RS, Joffe AR, Ross DB, Rebeyka IM, Western Canadian Complex Pediatric Therapies Follow-Up Group (2013) Risk factors for and outcomes of acute kidney injury in neonates undergoing complex cardiac surgery. J Pediatr 162:120-127.e121

13. Jetton JG, Askenazi DJ (2012) Update on acute kidney injury in the neonate. Curr Opin Pediatr 24:191-196

14. Alge JL, Arthur JM (2015) Biomarkers of AKI: A review of mechanistic relevance and potential therapeutic implications. Clin J Am Soc Nephrol. 10:147-155
15. Askenazi D (2012) Are we ready for the clinical use of novel acute kidney injury biomarkers? Pediatr Nephrol 27:1423-1425

16. Krawczeski CD, Goldstein SL, Woo JG, Wang Y, Piyaphanee N, Ma Q, Bennett M, Devarajan P (2011) Temporal relationship and predictive value of urinary acute kidney injury biomarkers after pediatric cardiopulmonary bypass. J Am Coll Cardiol 58:2301-2309

17. Zappitelli M, Washburn KK, Arikan AA, Loftis L, Ma Q, Devarajan P, Parikh CR, Goldstein SL (2007) Urine neutrophil gelatinase-associated lipocalin is an early marker of acute kidney injury in critically ill children: a prospective cohort study. Crit Care 11:R84

18. Askenazi DJ, Koralkar R, Hundley HE, Montesanti A, Parwar P, Sonjara S, Ambalavanan N (2012) Urine biomarkers predict acute kidney injury in newborns. J Pediatr 161:270-275.e271

19. Haase M, Bellomo R, Devarajan P, Schlattmann P, Haase-Fielitz A (2009) Accuracy of neutrophil gelatinase-associated lipocalin (NGAL) in diagnosis and prognosis in acute kidney injury: a systematic review and meta-analysis. Am J Kidney Dis 54:1012-1024

20. Haase M, Devarajan P, Haase-Fielitz A, Bellomo R, Cruz DN, Wagener G, Krawczeski CD, Koyner JL, Murray P, Zappitelli M, Goldstein SL, Makris K, Ronco C, Martensson J, Martling CR, Venge P, Siew E, Ware LB, Ikizler TA, Mertens PR (2011) The outcome of neutrophil gelatinase-associated lipocalin-positive subclinical acute kidney injury: a multicenter pooled analysis of prospective studies. J Am Coll Cardiol 57:1752-1761

21. Basu RK, Wong HR, Krawczeski CD, Wheeler DS, Manning PB, Chawla LS, Devarajan P, Goldstein SL (2014) Combining functional and tubular damage biomarkers improves diagnostic precision for acute kidney injury after cardiac surgery. J Am Coll Cardiol 64: 2753-2762

22. Basu RK, Zappitelli M, Brunner L, Wang Y, Wong HR, Chawla LS, Wheeler DS, Goldstein SL (2014) Derivation and validation of the renal angina index to improve the prediction of acute kidney injury in critically ill children. Kidney Int 85:659-667

23. Basu RK, Andrews A, Krawczeski C, Manning P, Wheeler DS, Goldstein SL (2013) Acute kidney injury based on corrected serum creatinine is associated with increased morbidity in children following the arterial switch operation. Pediatr Crit Care Med 14:e218 e224

24. Liu KD, Thompson BT, Ancukiewicz M, Steingrub JS, Douglas IS, Matthay MA, Wright P, Peterson MW, Rock P, Hyzy RC, Anzueto A, Truwit JD (2011) Acute kidney injury in patients with acute lung injury: impact of fluid accumulation on classification of acute kidney injury and associated outcomes. Crit Care Med 39:2665-2671

25. Ricci Z, Luciano R, Favia I, Garisto C, Muraca M, Morelli S, Di Chiara L, Cogo P, Picardo S (2011) High-dose fenoldopam reduces postoperative neutrophil gelatinase-associated lipocaline and cystatin C levels in pediatric cardiac surgery. Crit Care 15:R160

26. Selewski DT, Jordan BK, Askenazi DJ, Dechert RE, Sarkar S (2013) Acute kidney injury in asphyxiated newborns treated with therapeutic hypothermia. J Pediatr 162:725-729.e721 